\title{
Scaling laws for dielectric window breakdown in vacuum and collisional regimes
}

\author{
Y. Y. Lau, ${ }^{\text {a) }}$ J. P. Verboncoeur, ${ }^{\text {b) }}$ and H. C. Kim ${ }^{\text {b) }}$ \\ Department of Nuclear Engineering and Radiological Sciences, University of Michigan, Ann Arbor, \\ Michigan 48109-2104
}

(Received 18 October 2006; accepted 29 November 2006; published online 29 December 2006)

\begin{abstract}
The scaling laws for the initiation time of radio frequency (rf) window breakdown are constructed for three gases: Ar, Xe, and Ne. They apply to the vacuum, to the multipactor-triggered regime, and to the collisional rf plasma regime, and they are corroborated by computer simulations of these three gases over a wide range of pressures. This work elucidates the key factors that are needed for the prediction of $\mathrm{rf}$ window breakdown in complex gases, such as air, at various pressures. (C) 2006 American Institute of Physics. [DOI: 10.1063/1.2425025]
\end{abstract}

In high power microwave systems, dielectric window breakdown remains a major issue. ${ }^{1-3}$ The vacuum multipactor discharge, ${ }^{1,4-7}$ often considered a candidate which initiates window breakdown on the vacuum side, is an avalanche caused by secondary electron emissions from the dielectric window. In contrast to breakdown on the vacuum side, there have been few studies on collisional discharge on the air side of a dielectric window. ${ }^{3}$ At high gas pressures, the collision of electrons with the background gas may alter the multipactor discharge significantly, or even render multipactor irrelevant. ${ }^{8,9}$

In this letter, we construct the scaling laws for the initiation time of a discharge on a dielectric surface that is embedded in one of three gases: Ar, Ne, and Xe. The scaling laws are derived for the low and high pressure limits. Noble gases are chosen for this initial study for the simplicity in the chemistry; their ionization and other collision cross sections are well characterized and straightforward (compared with air). While our earlier simulations ${ }^{9}$ were restricted to Ar, the data revealed sufficient information for the construction of scaling laws for Ar and predictions for $\mathrm{Ne}$ and Xe. An understanding of the physical basis for the scaling laws for these simple gases, together with the corroboration with numerical simulations, which we shall demonstrate here, provides some guidance on the crucial data that would be required to make window-breakdown predictions for complex gases such as air. This consideration is clearly relevant for a specific high power microwave application, where the pressures and the compositions in the background gas may vary greatly. Analogous scaling laws in the vacuum and high pressure limits, together with just one or two simulation data points in the transition regime for this complex gas, can provide an immediate assessment, for instance, of whether a high power microwave pulse of a given pulse length would be subject to window breakdown.

An important parameter in window-breakdown studies is the discharge formation time $\tau$, in particular, for pulsed high power microwaves. ${ }^{3,10}$ In this letter, we define $\tau$ to be the time required for the number of electrons to multiply by a factor of $10^{8}$ from its initial value, as a result of ionization. That is, $e^{\langle\nu\rangle \tau}=10^{8}$, yielding

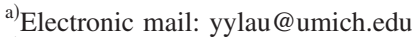

${ }^{\mathrm{b})}$ University of California, Berkeley, CA.
}

$$
\tau=\frac{18.4}{\langle\nu\rangle},
$$

where $\langle\nu\rangle$ is the average of the ionization rate $\nu_{i}$. By average, we refer to the average over the electron distribution, and over the constituents of background gas, if there are multiple species, assuming that the electron loss mechanisms have been accounted for. ${ }^{11}$ For a single species, the ionization rate is given by

$$
\nu_{i}=n \sigma v=3.22 \times 10^{9} \mathrm{~s}^{-1} \times\left(\frac{p}{1 \text { Torr }}\right) \times\left(\frac{\sigma v}{10^{-13} \mathrm{~m}^{3} / \mathrm{s}}\right) \text {, }
$$

where $n$ is the background density of the gas, $\sigma$ is the ionization cross section, and $v$ is the electron velocity. The last expression in Eq. (2) is in practical units, assuming that the gas pressure $p$ is related to the density $n$ at room temperature. It is clear from Eq. (2) that specifying $\nu_{i} / p$ is equivalent to specifying the rate $\sigma v$, which depends only on the energy of the electron for a given gas species.

In the low pressure regime, $1 / t_{F} \gg \omega \gg \nu_{c}$, where $t_{F}$ is the time of flight of a multipacting electron, $\omega$ is the frequency of the rf electric field, and $\nu_{c}$ is the collision frequency between this multipacting electron and the background gas. In this low pressure regime, a multipacting electron experiences primarily the vacuum rf electric field during its time of flight $t_{F}$, from its birth on the dielectric surface as a secondary electron to its impact onto the dielectric surface as the primary electron for the next generation of the secondary electrons, ${ }^{4}$ after its acceleration by the vacuum rf electric field during $t_{F}$. For pressures less than 1 Torr, this condition is largely satisfied. In such cases, the multipacting electron typically has an energy between $100 \mathrm{eV}$ and $2 \mathrm{keV},{ }^{8,9}$ for rf electric field of order of $1 \mathrm{MV} / \mathrm{m}$ and rf frequency below $10 \mathrm{GHz}$, regardless of the dielectric material.

An analytic scaling law may be constructed by first noting that for electron energy between $100 \mathrm{eV}$ and $2 \mathrm{keV}, \sigma v$ changes little, perhaps by a factor of 2 at the most. More importantly, the maximum value of $\sigma v$ lies within this range. For Ar, Xe, and $\mathrm{Ne}$, the maximum values of $\sigma v$ are, respectively, $1.8 \times 10^{-13}, 5.6 \times 10^{-13}$, and $6.2 \times 10^{-14} \mathrm{~m}^{3} / \mathrm{s}$. If we insert these maximum values of $\sigma v$ into Eq. (2) to obtain the maximum value of $\nu_{i}$, and for simplicity assume that the 


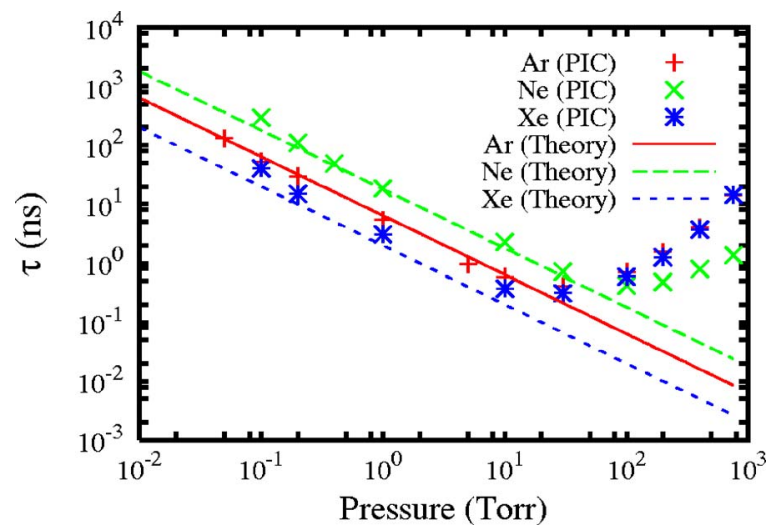

FIG. 1. (Color online) Simulation of the discharge formation time as a function of pressure for $\mathrm{Ar}, \mathrm{Ne}$, and $\mathrm{Xe}$. The straight lines are the scaling laws for the low pressure, multipactor-triggered regime [Eq. (3)].

average value of $\nu$ in Eq. (1) is half of this maximum value, ${ }^{11}$ then Eqs. (1) and (2) yield the following scaling laws for the discharge time:

$$
\begin{aligned}
& \tau=6.4 \mathrm{~ns} / p \text { (Torr) } \quad(\mathrm{Ar}), \\
& \tau=2 \mathrm{~ns} / p \text { (Torr) } \quad(\mathrm{Xe}), \\
& \tau=18 \mathrm{~ns} / p(\text { Torr }) \quad(\mathrm{Ne}) .
\end{aligned}
$$

Equations (3a)-(3c) are plotted in Fig. 1, where favorable comparison between these scaling laws and simulations may be noted.

The simulations were performed using the XPDP1 code, ${ }^{12}$ a standard particle-in-cell model ${ }^{13}$ with a Monte Carlo collision scheme. ${ }^{14}$ In the one-dimensional (1D) model with dielectric representing the microwave window, the plane-wave rf electric field is superimposed on the selfconsistent electrostatic space charge field; reflection and absorption of the wave by the plasma are neglected. Particles reaching the microwave window deposit charge and can stimulate secondary emission, modeled following Vaughan. ${ }^{15}$ The model includes a self-consistent kinetic development of the electron energy distribution function, which is strongly influenced by the collision cross sections.

In the high pressure regime where collisions dominate, $\omega \ll \nu_{c}$. The average kinetic energy of an electron, $W$, is lower than the ionization energy of the gas, $W_{i}$. In this case, we may begin with the approximate equation that governs the gain in the electron kinetic energy from the rf electric field in the collision-dominated regime, ${ }^{10}$

$$
\frac{d W}{d t}=\frac{e^{2} E_{0}^{2}}{2 m \nu_{c}}
$$

where $E_{0}$ is the amplitude of the $\mathrm{rf}$ electric field and $e$ and $m$ are, respectively, the electron charge and mass. Since Eq. (4) has not included the energy loss mechanism, for simplicity, we assume that the time $t_{i}$ required for the electron energy to reach $W_{i}$ is twice that inferred from Eq. (4). This then yields an average ionization rate, $\langle\nu\rangle=e^{2} E_{0}^{2} / 4 m\left\langle\nu_{c} W_{i}\right\rangle{ }^{11}$ We may use this expression of $\langle\nu\rangle$ in Eq. (1) to write the discharge time $\tau$ in practical units,

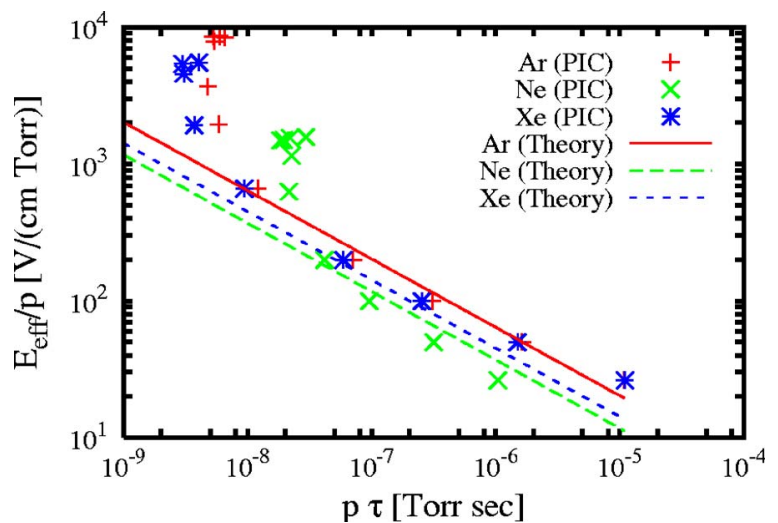

FIG. 2. (Color online) One-dimensional simulation of $E_{\mathrm{eff}} / p$ vs $p \tau$ for Ar, $\mathrm{Ne}$, and Xe. The straight lines are the scaling laws for the high pressure, collisional regime [Eq. (7)]. The data on the left, which align vertically, follow the low pressure scaling law, $p \tau \sim$ constant [Eq. (3)].

$$
\begin{aligned}
\tau= & 6.8 \times 10^{-12} \mathrm{~s} \times\left(\frac{\bar{W}_{i}}{10 \mathrm{eV}}\right)\left(\frac{\langle\sigma v\rangle_{c}}{10^{-13} \mathrm{~m}^{3 / \mathrm{s}}}\right) \\
& \times\left(\frac{p}{1 \text { Torr }}\right) \frac{1}{\left[E_{\mathrm{eff}} /(1 \mathrm{MV} / \mathrm{m})\right]^{2}},
\end{aligned}
$$

after using the relation $\nu_{c}=n \sigma_{c} v$ in terms of the electron collision cross section $\sigma_{c}$, and the relation between $n$ and $p$ [assuming room temperature, as in Eq. (2)]. In Eq. (5), $E_{\text {eff }}$ $=E_{0} / \sqrt{2}$ is the effective electric field in the collisiondominated regime, $\bar{W}_{i}$ is the average ionization energy of the background gas, and $\langle\sigma v\rangle_{c}$ is the electron collision rate averaged over the electron distribution function. Equation (5) shows that $\tau$ increases with $p$, and that it has a strong dependence on the rf electric field. Multiplying Eq. (5) by $p$, we may rewrite Eq. (5) as

$$
\begin{aligned}
\frac{E_{\text {eff }}}{p}\left(\frac{V}{\mathrm{~cm} \text { Torr }}\right)= & \frac{0.026}{\sqrt{(p \tau / \text { Torr s })}} \\
& \times \sqrt{\left(\frac{\bar{W}_{i}}{10 \mathrm{eV}}\right)\left(\frac{\langle\sigma v\rangle_{c}}{10^{-13} \mathrm{~m}^{3} / \mathrm{s}}\right)} .
\end{aligned}
$$

Equation (6) shows the primary dependence of $E_{\mathrm{eff}} / p$ on $(p \tau)^{-1 / 2}$ and a relatively weak dependence on the rf electric field and rf frequency, consistent with our simulations, as shown in Figs. 2 and 3.

A qualitative scaling law may also be constructed for the high pressure regimes by first noting that $\langle\sigma v\rangle_{c}$ does not change appreciably when the electron energy is in the vicinity of the ionization threshold. For simplicity, we assume that $\bar{W}_{i}$ in Eq. (6) is this ionization threshold, which is approximately equal to 16,12 , and $22 \mathrm{eV}$ for, respectively, $\mathrm{Ar}$, Xe, and Ne. For electron energy in the vicinity of this ionization threshold, the values of $\langle\sigma v\rangle_{\mathrm{c}}$ for these three gases are, respectively, $4 \times 10^{-13}, 2.4 \times 10^{-13}$, and $9.3 \times 10^{-14} \mathrm{~m}^{2} / \mathrm{s}$. We next insert these values of $\bar{W}_{i}$ and $\langle\sigma v\rangle_{c}$ into Eq. (6) to obtain the scaling laws for these three gases in the high pressure regime,

$$
\begin{aligned}
& E_{\text {eff }} / p[\mathrm{~V} /(\mathrm{cm} \text { Torr })]=0.064 / \sqrt{p \tau(\text { Torr } \mathrm{s})} \quad(\mathrm{Ar}), \\
& E_{\mathrm{eff}} / p[\mathrm{~V} /(\mathrm{cm} \text { Torr })]=0.045 / \sqrt{p \tau(\text { Torr } \mathrm{s})} \quad(\mathrm{Xe}),
\end{aligned}
$$




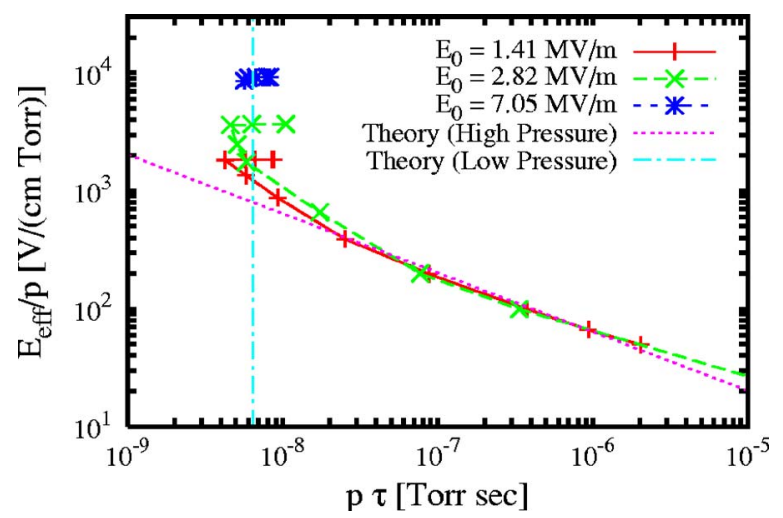

FIG. 3. (Color online) Pseudo-three-dimensional simulation of $E_{\text {eff }} / p$ vs $p \tau$ for Ar, at various values of rf electric field. The vertical straight line represents the low pressure scaling law [Eq. (3a)], $p \tau=6.4 \times 10^{-9}$ Torr s. The slanting straight line represents the high pressure scaling law [Eq. (7a)], $E_{\mathrm{eff}} / p[\mathrm{~V} /(\mathrm{cm}$ Torr $)]=0.064[p \tau(\text { Torr } \mathrm{s})]^{-1 / 2}$.

$$
E_{\mathrm{eff}} / p[\mathrm{~V} /(\mathrm{cm} \text { Torr })]=0.037 / \sqrt{p \tau(\text { Torr s })} \quad(\mathrm{Ne}) .
$$

Equations (7a)-(7c) are plotted in Fig. 2 which shows qualitative agreement with the simulation. The deviation between the scaling laws and the simulation results in Fig. 2 is due to the crude assumptions made on $\bar{W}_{i}$ and $\langle\sigma v\rangle_{c}$ that are used in Eq. (6). ${ }^{11}$

We should point out that the simulation data in Fig. 2 also exhibit the scaling laws for the low pressure regime. The data points on the left of each curve of Fig. 2 are aligned almost vertically, i.e., $p \tau \sim$ constant, in agreement with Eqs. (3a)-(3c) for the low pressure regime. These features are shared in both 1D and three-dimensional (3D) simulations, ${ }^{8}$ the latter including transverse mode structures and additional transverse electron loss ${ }^{11}$ which are absent in the 1D planewave model. Figure 3 shows the pseudo-3D Ar simulations ${ }^{9}$ at very different levels of $\mathrm{rf}$ electric fields (1.41-7.05 MV/m) using a $\mathrm{TE}_{10}$ mode but neglecting transverse space charge effects. In Fig. 3, the analytic scaling of Eq. (3a) is shown by the vertical line, whereas Eq. (7a) is shown by the straight line with a negative slope. Thus, these analytic scalings with the electric field, for the low and high pressure regimes, are corroborated with both 1D simulations (Fig. 2) and 3D simulations (Fig. 3).

The above study of simple gases suggests that, in the case of a complex gas, the scaling laws for the discharge formation time in the vacuum limit may be constructed from a knowledge of the average ionization rate, $\langle\sigma v\rangle$, for this complex gas for electron energy in the $100-2 \mathrm{keV}$ range [see Eq. (2)]. Assuming that the ionization rate of this complex gas is insensitive to the precise electron energy in this electron energy range, its rate may be evaluated at a single electron energy, and this rate then yields the scaling law in the low pressure regime, similar to Eqs. (3a)-(3c). In the high pressure regime, the scaling law for this complex gas may be obtained from the threshold ionization energy $W_{i}$, and the electron collision rate $\sigma_{c} v$ for electron energy at $W_{i}$ [see Eqs. (6) and (7)]. Once the scaling laws for the low pressure and high pressure regimes are thus constructed, a simulation at one pressure that lies in the transition region will then provide a ready estimate for the discharge formation time for all pressures for this complex gas.

This work was supported by AFOSR Cathode and Breakdown MURI04 Grant No. FA9550-04-1-0369, AFOSR STTR Phase II Contract No. FA9550-04-C-0069, and the Air Force Research Laboratory, Kirtland AFB.

${ }^{1}$ A. A. Neuber, L. Laurent, Y. Y. Lau, and H. Krompholz, in High Power Microwave Sources and Technologies, edited by R. J. Barker and E. Schamiloglu (IEEE Press, Piscataway, NJ, 2001), chap. 10, p. 325.

${ }^{2}$ D. Shiffler, G. Baca, T. Englert, M. D. Haworth, K. J. Hendricks, D. Henley, M. Sena, and T. Spencer, IEEE Trans. Plasma Sci. 26, 304 (1998).

${ }^{3}$ G. Edmiston, J. Krile, A. Neuber, J. Dickens, and H. Krompholz, IEEE Trans. Plasma Sci. 34, 1782 (2006).

${ }^{4}$ R. A. Kishek and Y. Y. Lau, Phys. Rev. Lett. 80, 193 (1998); R. A. Kishek, Y. Y. Lau, L. K. Ang, A. Valfells, and R. M. Gilgenbach, Phys. Plasmas 5, 2120 (1998).

${ }^{5}$ R. A. Rimmer, Ph.D. dissertation, Lancaster University, Lancaster, UK, 1998.

${ }^{6}$ A. Sazontov, V. Semenov, M. Buyanova, N. Vdovicheva, D. Anderson, M. Lisak, J. Puech, and L. Lapierre, Phys. Plasmas 12, 093501 (2005).

${ }^{7}$ H. C. Kim and J. P. Verboncoeur, Phys. Plasmas 12, 123504 (2005).

${ }^{8}$ H. C. Kim and J. P. Verboncoeur, Phys. Plasmas 13, 123506 (2006).

${ }^{9}$ H. C. Kim, J. P. Verboncoeur, Y. Chen, and Y. Y. Lau, Proceedings of the Seventh IEEE International Vacuum Electronics Conference, Monterey, CA (IEEE, Piscataway, NJ, 2006), p. 359.

${ }^{10}$ D. Anderson, M. Lisak, and T. Lewin, J. Appl. Phys. 56, 1414 (1984).

${ }^{11}$ The electron loss and the evolution of electron distribution functions are not explicitly considered in the analytic theory. Thus, we simply assume that $\langle\nu\rangle$ in Eq. (1) is reduced by the same factor $\frac{1}{2}$ in its estimate for both low pressure and high pressure scalings.

${ }^{12}$ J. P. Verboncoeur, M. V. Alves, V. Vahedi, and C. K. Birdsall, J. Comput. Phys. 104, 321 (1993).

${ }^{13}$ C. K. Birdsall and A. B. Langdon, Plasma Physics via Computer Simulation (McGraw-Hill, New York, 1985).

${ }^{14}$ V. Vahedi and M. Surendra, Comput. Phys. Commun. 87, 179 (1995).

${ }^{15}$ J. R. M. Vaughan, IEEE Trans. Electron Devices 40, 830 (1993). 